\title{
Antilocalization Effect on Photo-Generated Carriers in Semi-Insulating Gaas Sample
}

\author{
Emilson Ribeiro Viana ${ }^{\text {a }}$,Geraldo Mathias Ribeiro ${ }^{\text {a }}$, Alfredo Gontijo de Oliveira ${ }^{\text {a }}$, \\ Marcelos Lima Peres ${ }^{\mathrm{b} *}$, Rero Marques Rubinger ${ }^{\mathrm{b}}$, Carla Patrícia Lacerda Rubinger ${ }^{\mathrm{b}}$ \\ ${ }^{a}$ Departamento de Física, ICEx, Universidade Federal de Minas Gerais - UFMG, \\ CP 702, CEP 30123-970, Belo Horizonte, MG, Brasil \\ 'Instituto de Ciências, Departamento de Física e Química, Universidade Federal de Itajubá - UNIFEI, \\ CP 50, CEP 37500-903, Itajubá, MG, Brasil
}

Received: September 14, 2011; Revised: April 6, 2012

\begin{abstract}
Magnetoresistance measurements were performed on an illuminated semi-insulating GaAs sample with intrinsic deep level defects. Electrical carriers were photo-generated under light excitation and positive magnetoresistance for $B<0.2 \mathrm{~T}$ was observed in the whole range of temperatures measured (220-315 K). Using the model developed by H. Fukuyama and K. Hoshino, we interpreted the positive magnetoresistance as mainly caused by weak antilocalization effects over hole carriers for $T>240 \mathrm{~K}$. The high disorder originated from the low temperature growth of the sample leads to the strong localization of carriers and gives rise to the positive magnetoresistance observed at temperatures as high as room temperature.
\end{abstract}

Keywords: semi-insulating GaAs, deep-level defects, antilocalization

\section{Introduction}

Electron transport in disordered systems has been widely studied over the past 30 years ${ }^{1-3}$ and is a powerful tool for probing electron-electron interactions ${ }^{4}$ and weak localization phenomenon ${ }^{5}$. For 2D systems, low field positive magnetoresistance has been observed on GaAs based structures and it is attributed to the spin-orbit coupling interaction which could be originated from the electric field of the atomic nucleus or related to the particular characteristics of crystalline and band structure of the solid $^{6-10}$. Nevertheless, few studies about spin-orbit coupling interaction have been reported on $3 \mathrm{D}$ semiconductor thin films ${ }^{6,11,12}$. One method to determine the strength of the spin-orbit coupling is through the weak anti-localization effect (suppression of weak localization due spin-orbit interactions $)^{1}$. One can use the models that predict the contribution to the magnetoconductivity due to this effect and obtain the inelastic and spin-orbit scattering times and verify how they respond to the change of temperature or carrier concentration ${ }^{8-10}$.

In this work, we report on the positive magnetoresistance measured on an illuminated GaAs sample in the temperature region of 220-315 K with intrinsic deep level defects. For the temperatures higher than $240 \mathrm{~K}$, the positive magnetoresistance indicates the presence of spin-orbit coupling over the hole carriers that diffuse in a highly disordered system. The disorder is originated during the growth process where the GaAs material extremely rich in intrinsic deep level defects is obtained by low temperature molecular beam epitaxy (LT- MBE) growth ${ }^{13}$. This material is used in applications on the development of high quality

*e-mail: marcelos@unifei.edu.br insulating substrates after applying post-growth annealing ${ }^{14}$ and there is an academic interest in the properties of the main intrinsic deep levels found in such materials, i.e., the arsenic anti-site $\left(\mathrm{As}_{\mathrm{Ga}}\right)$, a well-known mid-gap donor ${ }^{14}$, that can be found in concentrations that reach up to $10^{26} \mathrm{~m}^{-3}$ as in a typical LT-MBE sample grown. In such system, the carriers can be promoted to the bands from the $\mathrm{As}_{\mathrm{Ga}}$ at temperatures above the room temperature or generated under illumination with an infrared GaAs LED at any temperature. In darkness, the $\mathrm{As}_{\mathrm{Ga}}$ deep levels act as recombination centers, removing most band carriers leaving only hopping conduction. Thus, in darkness, we can identify the hopping contribution to the magnetoresistive effects while under illumination we can observe the band contribution. Since the localization radius of electronic wave functions is very short (i.e., of the order of $10 \AA$ ), no magnetoresistive effects due the wave function shrinkage will be observed for magnetic fields below $1 \mathrm{~T}$. As will be shown, this is confirmed by our measurements. Thus, all magnetoresistive effects reported here were obtained under illumination and consist in effects related to scattering of band carriers by randomly distribute $\mathrm{As}_{\mathrm{Ga}}$ anti-sites. In addition, the combination/recombination process via $\left(\mathrm{As}_{\mathrm{Ga}}\right)$ defects, subsequent to the carrier excitation, is different for electrons and holes under illumination conditions and its rate is temperature dependent. This property is also taken in account during our analysis and we found that holes are the majority carriers for the conductivity process in this sample. We analyzed the magnetoresistance curves using the model of Fukuyama and Hoshino ${ }^{15}$ considering localization/anti-localization effects and obtained the inelastic and spin-orbit scattering times as a function of the temperature. Since the quantitative description of the 
spin-orbit interaction in a given material is the main key for new advances in spintronics field ${ }^{16}$, we expect that our work could shed some light on the possibility of using the spin-orbit interaction for the development of room temperature spintronic devices.

\section{Material and Methods}

The samples were grown on (1 00 ) SI liquid encapsulated Czochralski GaAs substrates, in a RIBER $2300 \mathrm{MBE}$ system. After the standard desorption procedures, a $0.21 \mu \mathrm{m}$ thick buffer layer was grown at $610{ }^{\circ} \mathrm{C}$ under an As overpressure of $3.2 \times 10^{-5}$ Torr and 0.81 monolayer/s. After that, the substrate temperature was ramped down at $10{ }^{\circ} \mathrm{C} / \mathrm{min}$ to the final desired temperature while growing. After reaching the set temperature of $265^{\circ} \mathrm{C}$, a $2.4 \mu \mathrm{m}$ thick GaAs epilayer was grown. The resistivity measurements were done using indium parallel bars contacts on samples that were cut into $1 \times 2 \mathrm{~mm}^{2}$. The distance between the bars was $0.5 \mathrm{~mm}$. This geometry was necessary in order to measure the electrical resistance in dark conditions. After depositing the contacts and attaching gold wires with a soldering station at a temperature of $250{ }^{\circ} \mathrm{C}$ the samples were not post-annealed. The measurements were carried out in the low electric field regime, i.e., in the ohmic regime that was identified from I(V) conductivity measurements, carried out from negative to positive bias, giving us a certainty about the linearity of the contact resistance in all the measured samples. We prepared at least seven pieces of the studied sample from different parts of the wafer and for a same wafer the results did not vary significantly. For carrying out the resistivity measurements, we used a Keithley automated Hall System model 7065, the standard current source and the requested meters. All contacts between the cryostat and the Hall system were done with triaxial cables in guarded mode measurements to avoid parasitic capacitance. Inside the cryostat, $2 \mathrm{~mm}$ diameter 50 Teflon insulated coaxial cables were used.

\section{Results}

The sample was illuminated by a GaAs infrared LED and the photon flux is linearly dependent on the current through the LED. Thus, for sake of simplicity, we will refer to the illumination intensity by the corresponding current from here on. In Figure 1 we present the electrical resistance $R$ as a function of the temperature for different illumination intensities. For dark conditions, i.e., with $0 \mathrm{~mA}, R$ increases as the temperature decreases while under illumination it decreases below $240 \mathrm{~K}$ when the illumination varies from $0 \mathrm{~mA}$ to $10 \mathrm{~mA}$. For illumination with current above $10 \mathrm{~mA}$, the electrical resistance oscillates as the temperature decreases between $260 \mathrm{~K}$ and $240 \mathrm{~K}$ and starts to decrease for temperatures below $240 \mathrm{~K}$. In Figure $2 \mathrm{a}$ we present the normalized $R$ as a function of the magnetic field with different illumination at $T=300 \mathrm{~K}$. Through this figure we observe that no magnetoresistive effect is observed in dark conditions $(0 \mathrm{~mA})$ (e.g. the slight oscillations have no physical meaning) while a negative magnetoresistance can be observed for illumination with current of $5 \mathrm{~mA}$. For illuminations with current higher than $5 \mathrm{~mA}$, the curves exhibit positive peaks followed by negative magnetoresistance as the magnetic field increases. In Figure $2 \mathrm{~b}$, we show the normalized $R$ as a function of the magnetic field for different temperature values at a fixed illumination with current of $40 \mathrm{~mA}$. The amplitude of the peaks increases as the temperature decreases in the region for $B<0.2 \mathrm{~T}$ while a parabolic behavior is observed for $B>0.4 \mathrm{~T}$ due to the classical orbital effect (see inset). In Figure $2 \mathrm{~b}$ some curves were omitted in order to simplify the view.

\section{Discussion}

The electron-hole combination/recombination process via deep level defects is temperature dependent and its rate is different for conduction and valence bands under illumination conditions. The temperature for which $R$ starts to decrease observed in Figure 1 could be related to the temperature dependence of the combination/recombination rates in the conduction/valence bands. For example, if the hole recombination time increases as the temperature decreases the hole remains longer in the valence band leading to an enhancement of the conductivity in that channel. This also implies that the ratio between electrons and holes is temperature dependent. In fact, $n$ - to $p$-type transition is possible for illuminated GaAs samples ${ }^{17}$. In Table 1 we observe that the carriers mobility obtained when the sample is illuminated is about $0.119 \mathrm{~m}^{2} / \mathrm{Vs}$ at $T=300 \mathrm{~K}$. This value is lower than the value found in the literature for electrons $\left(\sim 0.850 \mathrm{~m}^{2} / \mathrm{Vs}\right)$ and higher than the value for holes $\left(\sim 0.04 \mathrm{~m}^{2} / \mathrm{Vs}\right)$. Deriving the carriers mobility considering a multi-channel mechanism ${ }^{18}$, we found that the effective mobility decreases if the ratio $p / n$ increases and a value of $p / n=10^{2}$ would lead to the mobility values shown in Table 1. This indicates that holes are the majority carriers in this sample. In addition, using the parameters

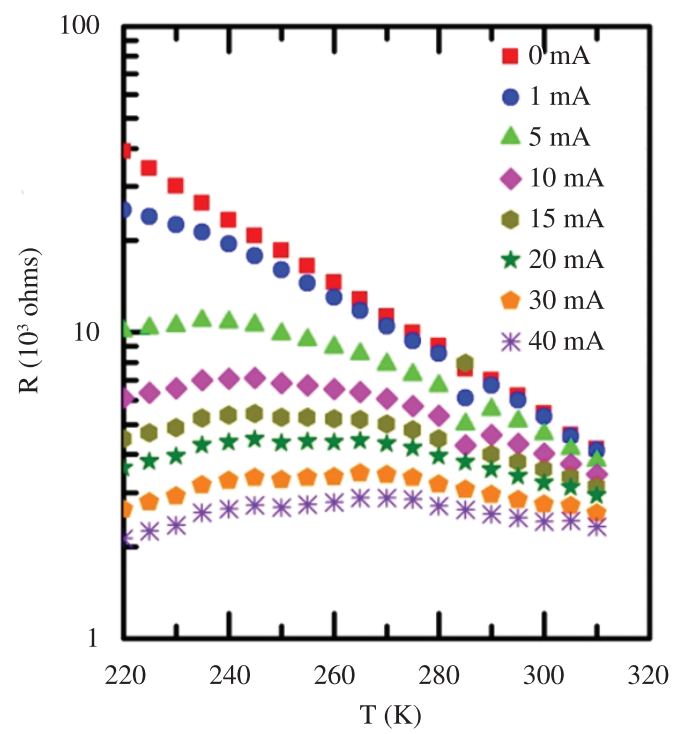

Figure 1. Electrical resistance as a function of the temperature for different illumination intensities. The temperature varied from $220 \mathrm{~K}$ up to $315 \mathrm{~K}$ while the illumination intensity increases with current ranging from $0 \mathrm{~mA}$ up to $40 \mathrm{~mA}$. 


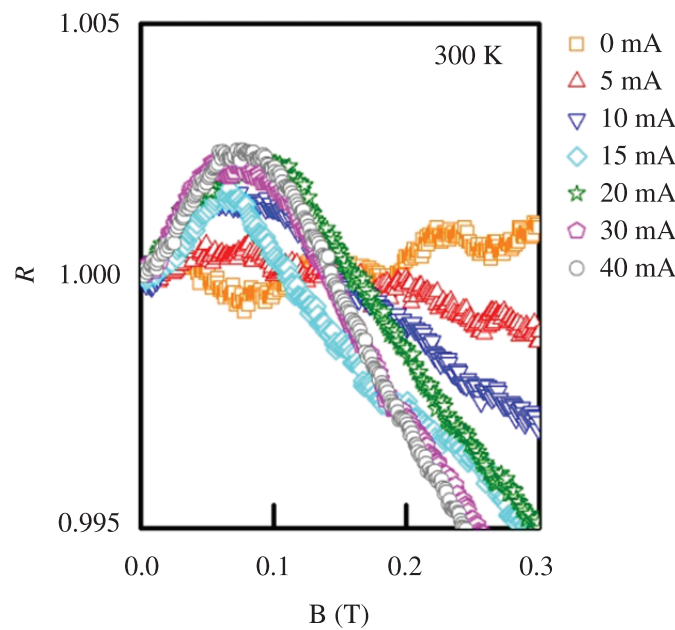

(a)

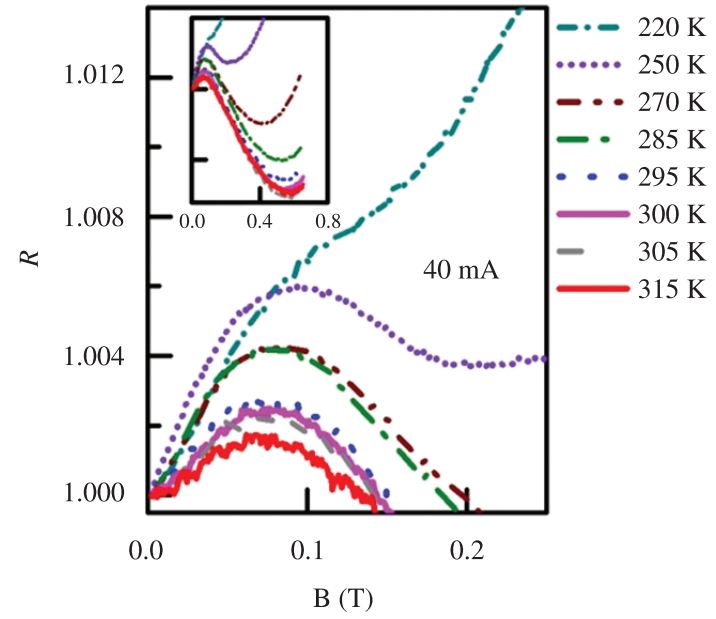

(b)

Figure 2. a) Normalized electrical resistance $R$ at $300 \mathrm{~K}$ for different illumination intensities. The peaks observed for $B<0.2 \mathrm{~T}$ scale with the light intensities which varies with the electrical current through the LED. b) Normalized electrical resistance for different temperatures at $40 \mathrm{~mA}$ through the LED. The peaks are also temperature dependent. The inset shows the curves for higher magnetic fields where the classical parabolic behavior of magnetoresistance can be observed.

Table 1. Temperature $(T)$, carrier mobility $(\mu)$, the parameter $C$, and the hole concentration $p$.

\begin{tabular}{cccc}
\hline$T(\mathbf{K})$ & $\boldsymbol{\mu}\left(\mathbf{m}^{\mathbf{2}} / \mathbf{V s}\right)$ & $\mathbf{C}\left(\times \mathbf{1 0} \mathbf{0}^{-\mathbf{1 4}} \mathbf{m}^{\mathbf{2}}\right)$ & $t$ \\
\hline 315 & 0.102 & 4.30 & 0.575 \\
305 & 0.110 & 4.35 & 0.539 \\
300 & 0.119 & 4.00 & 0.528 \\
295 & 0.135 & 4.80 & 0.506 \\
290 & 0.162 & 5.50 & 0.455 \\
285 & 0.173 & 5.70 & 0.450 \\
280 & 0.180 & 5.40 & 0.443 \\
270 & 0.216 & 5.40 & 0.434 \\
250 & 0.318 & 5.50 & 0.405 \\
\hline
\end{tabular}

given in Rubinger et al. ${ }^{19}$, we calculate the band to impurity recombination time $\left(\tau_{R}\right)$ for electrons and holes taking the $\mathrm{As}_{\mathrm{Ga}}$ defects concentration about $10^{25} \mathrm{~m}^{-3[20]}$. We found that the recombination time for holes $\left(\tau_{R}^{h}\right)$ is about $(5-6) \times 10^{-9} \mathrm{~s}$ between $315 \mathrm{~K}$ and $220 \mathrm{~K}$ while for electrons $\left(\tau_{R}^{e}\right)$ it is about $(5-12) \times 10^{-12} \mathrm{~s}$ in the same range of temperature.The elastic scattering time varies from $2.89 \times 10^{-13} \mathrm{~s}$ to $9 \times 10^{-13} \mathrm{~s}$ in the range of $315 \mathrm{~K}$ to $250 \mathrm{~K}$. Hence, we observe that $\tau_{R}^{h}$, $\tau_{R}^{6} \gg>$ but also that $\tau_{R}^{h} \gg>\tau_{R}^{6}$. This analysis also indicates that the holes are the majority carriers for the band conduction processes in the range of temperature analyzed, i.e., the condition $\tau_{R} \gg \tau$ indicates that the carrier has time enough to diffuses through the band before recombines back to the impurity level.

The positive magnetoresistance observed on Figure $2 \mathrm{a}, \mathrm{b}$ in the region of low magnetic fields $(B<0.2 \mathrm{~T})$, can have different origins. For insulator materials, where the hopping conduction is the main conduction mechanism, the shrinkage of the electronic wave functions caused by the application of a magnetic field could cause such positive magnetoresistance ${ }^{21}$. In this case, the carrier becomes more localized in a certain space region, increasing the electrical resistance as the magnetic field increases. However, no positive magnetoresistance is observed under dark conditions according to Figure $2 \mathrm{a}$ where the hopping conduction mechanism dominates in this sample. In addition, the magnetic length $l_{B}\left(\sim 10^{-7} \mathrm{~m}\right)$ is always larger than the mean free path $l$ that is around $\sim 10^{-8} \mathrm{~m}$, in the range $220-315 \mathrm{~K}$, indicating that the confinement due the magnetic field is negligible.

On the other hand, since the magnetoresistance presented in Figure 2 exists only under illumination, one can suggest that such effect could be caused by the influence of the magnetic field on the electron/hole recombination time. In fact, recombination can be spin-dependent and hence, magnetic field dependent. If the relative spins alignment is destroyed by a magnetic field, the recombination rate $\left(1 / \tau_{\mathrm{R}}\right)$ increases leading to an increase of the resistivity ${ }^{22}$. However, a spin-dependent conductivity change of only $\Delta \sigma / \sigma_{0} \sim 0.1 \%$ has been observed at cryogenic temperatures and under the application of an external magnetic field in $\mathrm{Si}$ or $\mathrm{Ge}^{22,23}$. The effect of the magnetic field on spin dependent recombination is more pronounced if the sample is illuminated by circularly polarized light ${ }^{24}$. GaAsN samples illuminated by circularly polarized light can exhibit photo-conductive variations of $40 \%{ }^{25}$. However, such effect has not been observed in undoped GaAs samples ${ }^{25}$. In addition, only $0.05 \%$ of the light applied on our sample was found to be circularly polarized which is not sufficient to cause the magnetoresistance profiles reported here.

The weak antilocalization effect is another physical phenomenon that could give rise to the positive magnetoresistance observed in Figure 2a, b. Weak localization is a quantum effect that arises from the quantum interference between the electronic wave functions moving in the same path but in opposite directions. If the electronic wave functions have the same phase, the interference is constructive leading to a spatial localization of the electrons. This effect can be observed as a negative magnetoresistance 
(positive magnetoconductance) if an external magnetic field is applied to the system ${ }^{1}$. If the spin-orbit coupling in not negligible as compared to other possible interaction mechanism, the interference is destructive and the localization is suppressed leading to the effect called weak antilocalization which can be detected as a positive magnetoresistance (negative magnetoconductance) ${ }^{1}$. In this case, experimental data can be analyzed using the Fukuyama-Hoshino model and the spin-orbit coupling strength can be obtained ${ }^{15}$.

Recently, the Fukuyama-Hoshino theory has been successfully applied to GaAs low temperature MBE layers heavily doped with $\mathrm{Si}$ and $\mathrm{Be}$ where positive and negative magnetoresistance was observed as in our case ${ }^{12}$. Also, in Jung et al. ${ }^{12}$, they attributed the localization effects observed at temperatures as high as room temperature to the strongly localized carriers due to the presence of deep level impurities. Under such circumstances the localization effect can be observed since the carrier mean free path is shorter than the phase relaxation length. The difference from the case presented in Jung et al. ${ }^{12}$ to the present work is that the carriers in the sample studied here are photo-generated instead of excited from shallow donors and acceptors. According to the Fukuyama-Hoshino model, the contribution to the magnetoconductivity due to the localization effects is given by the following equation:

$$
\begin{aligned}
& \frac{\Delta \sigma(B, T)}{A}=\sqrt{h} F\left(\frac{1+t}{h}\right)+\frac{1}{2} \sqrt{\frac{h}{1-\gamma}}\left\{F\left(\frac{t_{+}}{h}\right)-F\left(\frac{t_{-}}{h}\right)\right\}- \\
& \frac{1}{\sqrt{1-\gamma}}\left(\sqrt{t_{-}}-\sqrt{t_{+}}\right)+\sqrt{t}-\sqrt{t+1}
\end{aligned}
$$

where

$$
A=\frac{\sqrt{3} e^{2}}{2 \pi^{2} \hbar C^{\frac{1}{2}}}, \quad h=\left(\frac{B e}{3 \hbar}\right) C, \quad t=\frac{\tau_{s o}}{4 \tau_{i}}
$$

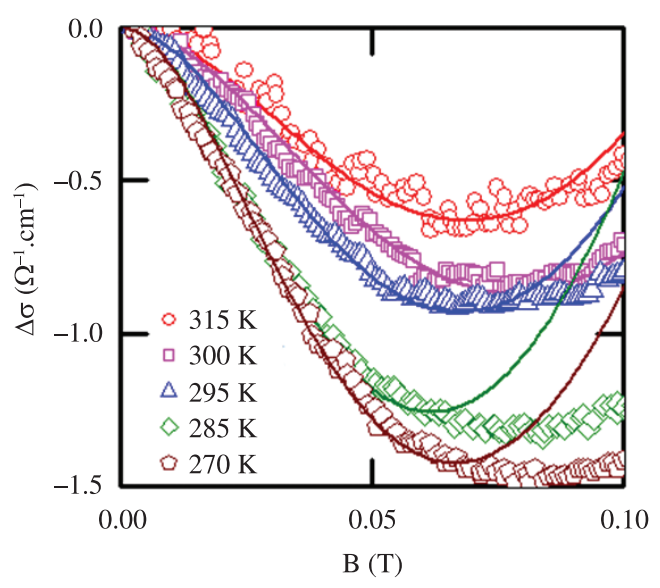

(a)

$$
\gamma=(k h)^{2}, t_{ \pm}=t+\frac{1}{2}(1 \pm \sqrt{1-\gamma})
$$

$C=l^{2} \tau_{s o} / \tau$, where $l$ is the mean free path, $\tau, \tau_{i}$ and $\tau_{s o}$ are the elastic, inelastic and spin-orbit scattering times, respectively, $F(x)$ is Kawabata's function and $k=\frac{3}{8}\left(m^{*} / m_{o}\right)\left|g^{*}\right| / \varepsilon_{F} \tau$.

According to Equation 1, the model also requires that $\gamma<1$. This condition is well satisfied for magnetic fields up to $\sim 0.2 \mathrm{~T}$. As told before, we consider that the holes are the majority carries in the range of $220 \mathrm{~K}$ to $315 \mathrm{~K}$. Particularly, the spin-orbit interactions are expected to be stronger in $p$-type materials and, in fact, this has been observed over different systems ${ }^{10,26,27}$. This fact is due to the $p$-like symmetry of the states at the top of the valence ${ }^{7}$. In addition, the $p$-like symmetry of hole states ensures that they are weakly coupled to nuclear spins, which could provide long spin coherence times. For the fitting procedure, we use the quantities $C, t$ and $k$ as fitting parameters and plotted the experimental data presented in Figure $2 \mathrm{~b}$ as $\Delta \sigma$ in order to compare with the theoretical calculations.

The fitting results are presented in Figure $3 \mathrm{a}$ for $0<B<0.1 \mathrm{~T}$ at the temperatures of $270,285,295,300$ and $315 \mathrm{~K}$ (the number of curves was reduced in order to clarify the visualization). Through this figure, one observes that the experimental curves are well described by the theoretical model for magnetic fields below $0.1 \mathrm{~T}$. It is also clear that the fitting quality reduces for temperatures below $270 \mathrm{~K}$. The values of the parameters $C$ and $t$ are presented in Table 1 together with the carriers mobility values which were calculated considering the classical behavior at high magnetic fields $\left(\Delta \mathrm{R} / \mathrm{R}_{0} \sim \mu^{2} \mathrm{~B}^{2}\right.$, where $\mu$ is the mobility) while the values of $\mathrm{k}$ did not vary considerably and were kept constant $(\mathrm{k}=0.2)$. In Figure $3 \mathrm{~b}$, we plot the temperature dependence of the $\mathrm{t}$ parameter presented in

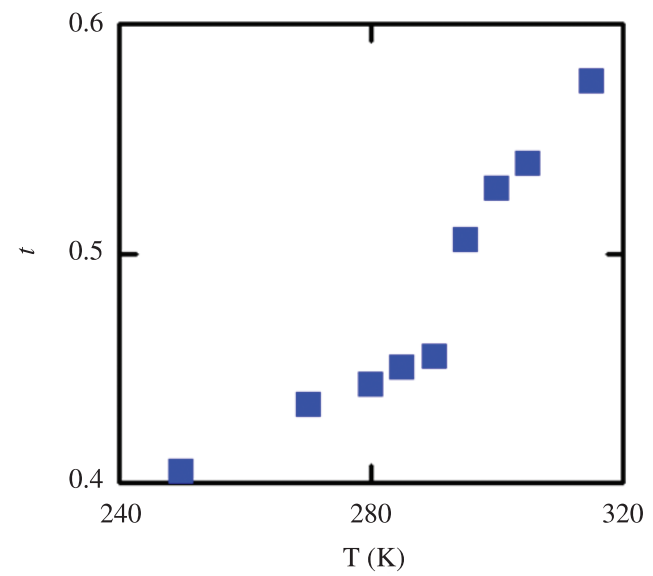

(b)

Figure 3. a) Magnetoconductivity correction according to the theoretical model (solid lines) as a function of the temperature together with the experimental points for a GaAs sample with $40 \mathrm{~mA}$ through the GaAs LED. The temperature varied from $220 \mathrm{~K}$ up to $315 \mathrm{~K}$. For sake of simplicity, some curves were omitted. b) Temperature dependence of the $t=\frac{\tau_{s o}}{4 \tau_{i}}$ parameter presented in Table 1 . According to this figure, $t$ decreases as temperature decreases indicating that spin-orbit coupling becomes more effective than the inelastic scattering mechanism. 
Table 1. According to this figure, $t$ decreases as temperature decreases indicating that spin-orbit coupling becomes more effective than the inelastic scattering mechanism. This is in accordance with the increasing of the positive peaks observed in Figure $2 \mathrm{a}$, b or the corresponding negative magnetoconductance observed in Figure 3 a.

It is still possible to estimate the value of $\tau_{\text {so }}$ rewriting $C$ relation as $\tau_{s o}=C \tau / l^{2}$. From the carriers mobility one can derive the elastic scattering time, i.e., $\tau=m_{h} \mu / e$, where $m_{h}$ is the hole effective mass $\left(\sim 0.5 \times m_{0}\right)$. The mean free path can be calculated from $l=v_{\mathrm{F}} \tau$, where $v_{F}=\hbar\left(3 \pi^{2} p\right)^{1 / 3} / m_{h}$ is the Fermi velocity. Considering a parabolic approximation, the values of the carriers concentration $p$ can be derived substituting the Fermi energy $\left(\varepsilon_{F}=\hbar^{2}\left(3 \pi^{2} p\right)^{2 / 3} / 2 m_{h}\right)$, in the $k$ relation above giving $p=\left[3 m_{h}^{2} 2 g / 8 m \tau \hbar\left(3 \pi^{2}\right)^{2 / 3} k\right]^{3 / 2}$. Thus, for $T=315 \mathrm{~K}$ and using the data presented in Table 1 we estimated $\tau_{\mathrm{so}} \sim 4.9 \times 10^{-11} \mathrm{~s}$.

This value agrees with the already reported results for Bulk GaAs samples at room temperature ${ }^{28}$ and for those obtained for GaAs quantum wells at low temperatures ${ }^{8}$.

\section{References}

1. Bergmann G. Weak Localization in Thin Films. Physics Reports. 1984; 107(1):1-58. http://dx.doi. org/10.1016/0370-1573(84)90103-0

2. Lin BJF, Paalanen MA, Gossard AC and Tsui DC. Weak localization of two-dimensional electrons in GaAs-AlGa1-As heterostructures. Physical Review B. 1984; 29:927-934. http:// dx.doi.org/10.1103/PhysRevB.29.927

3. Lin JJ and Bird JP. Recent experimental studies of electron dephasing in metal and semiconductor mesoscopic structures. Journal of Physics: Condensed Matter. 2002; 14(18):R501. http://dx.doi.org/10.1088/0953-8984/14/18/201

4. Altshuler BL, Aronov AG and Lee PA. Interaction effects in disordered fermi systems in two dimensions. Physical Review Letters. 1980; 44:1288-1291. http://dx.doi.org/10.1103/ PhysRevLett.44.1288

5. Anderson PW, Abrahms E and Ramakrishnan TV. Possible explanation of nonlinear conductivity in thin-film metal wires. Physical Review Letters. 1979; 43:718-720. http://dx.doi. org/10.1103/PhysRevLett.43.718

6. Gilbertson AM, Newaz AKM, Chang W-J, Bashir R, Solin SA and Cohen LF. Dimensional crossover and weak localization in a $90 \mathrm{~nm} n$-GaAs thin films. Applied Physics Letters. 2009; 95:012113-6. PMid:19668705 PMCid:2723832. http://dx.doi.org/10.1063/1.3176968

7. Grbic B, Leturcq R, Ihn T, Ensslin K, Reuter D and Wieck AD. Strong spin-orbit intereactions in carbon doped $p$-type GaAs heterostructures. Physica E. 2008; 40:2144-2146. http://dx.doi. org/10.1016/j.physe.2007.10.037

8. Desrat W, Maude DK, Wasilewski ZR, Airey R and Hill G. Dresselhaus spin-orbit coupling in a symetric (100) GaAs quantum well. Physical Review B. 2006; 74:193317-21. http:// dx.doi.org/10.1103/PhysRevB.74.193317

9. Cabañas S, Schapers TH, Thillosen N, Kaluza N, Guzenko VA and Hardtdegen $\mathrm{H}$. Suppression of weak antilicalization in an AlxGa1-xN/GaN two-dimensional electron gas by an in-plane magnetic field. Physical Review B. 2007; 75:195329-34. http:// dx.doi.org/10.1103/PhysRevB.75.195329

\section{Conclusions}

We have reported on magnetoresistance measurements performed on an illuminated semi-insulating GaAs sample with intrinsic deep level defects. Positive and negative magnetoresistances were observed for $B<0.2 \mathrm{~T}$ in the whole range of temperatures measured (220-315 K). For $T>240 \mathrm{~K}$ we explain the positive magnetoresistance curves as a manifestation of the weak antilocalization effect which is a consequence of the spin-orbit coupling interaction over the hole carriers. The Fukuyama-Hoshino model gives a good description of the experimental curves according to the antilocalization theory. From the analysis, we estimated the value of the spin-orbit scattering time at room temperature and found that it is close to the values reported before for bulk and GaAs quantum wells.

\section{Acknowledgements}

The authors acknowledge the Brazilian Agencies CNPq, CAPES and FAPEMIG for financial support.

10. Papadakis SJ, De Poortere EP, Manoharan HC, Yau JB, Shayegan $\mathrm{M}$ and Lyon SA. Low-field magnetoresistance in GaAs twodimensional holes. Physical Review B. 2002; 65:245312-9. http://dx.doi.org/10.1103/PhysRevB.65.245312

11. Look DC. Molecular beam epitaxial GaAs grown at low temperatures. Thin Solid Films. 1993; 231:61-73. http://dx.doi. org/10.1016/0040-6090(93)90703-R

12. Jung DW, Noh JP, Islam AZMT and Otsuka N. Enhanced electron-electron interaction in GaAs layers doped with high concentrations of donor and acceptor impurities. Journal of the Physical Society of Japan. 2008; 77:074721-8. http://dx.doi. org/10.1143/JPSJ.77.074721

13. Lin TC and Okumura T. Characterization of annealed low-temperature GaAs layers grown by molecular beam epitaxy with n-i-n structure. Japanese Journal of Applied Physics. 1996; 35:1630-1636. http://dx.doi.org/10.1143/ JJAP. 35.1630

14. Jost W, Kunzer M, Kaufmann U, Kohler K, Schneider J and Alt HC. AsGa antisite defects in LT GaAs as studied by magnetic resonance and magneto-optical techniques. Semiconductor Science and Technology. 1992; 7:1386-1389. http://dx.doi. org/10.1088/0268-1242/7/11/017

15. Fukuyama $\mathrm{H}$ and Hoshino K. Effecto of spin-orbit interaction on magnetoresistance in the weakly localized regime of three-dimensional disordered systems. Journal of the Physical Society of Japan. 1981; 50:2131-2132. http://dx.doi. org/10.1143/JPSJ.50.2131

16. Datta $\mathrm{S}$ and Das B. Electronic analog of the electro-opitc modulator. Applied Physics Letters. 1990; 56:665-667. http:// dx.doi.org/10.1063/1.102730

17. Da Silva MIN, De Oliveira AG, Ribeiro GM, Rubinger RM, Corrêa JA and Baeta MMV. EL2-like metastable defect and the $n$ - to $p$-type transition in silicon planar-doped GaAs. Journal of Applied Physics. 1997; 82:3346-3350. http://dx.doi. org/10.1063/1.365645

18. Corrêa JAF, De Oliveira AG, Da Silva MIN, Moreira MVB, Ribeiro GM and Chacham H. Temperature dependence of the 
equilibrium Hall concentration in silicon planar-doped GaAs samples. Solid State Communications. 1996; 98:1063-1068. http://dx.doi.org/10.1016/0038-1098(96)00146-9

19. Rubinger RM, Bezerra JC, Chagas EF, González JC, Rodrigues WN, Ribeiro GM et al. Thermally stimulated current spectroscopy on silicon planar-doped GaAs samples. Journal of Applied Physics. 1998; 84:3764-3769. http://dx.doi. org/10.1063/1.368588

20. Krambrock K, Linde M, Spaeth JM, Look DC, Bliss $\mathrm{D}$ and Walukiewicz W. Arsenic antisite-related defects in low-temperature MBE grown GaAs. Semiconductor Science and Technology. 1992; 7:1037-1041. http://dx.doi. org/10.1088/0268-1242/7/8/002

21. Shklowskii BI and Efros AL. Electronic properties of Doped Semiconductors. Springer-Verlag Berlin; 1984. v. 45, 210 p. Springer Series of Solid State Science.

22. Lepine DJ. Spin-Dependent Recombination on Silicon Surface. Physical Review B. 1972; 6:436-441. http://dx.doi.org/10.1103/ PhysRevB.6.436

23. Dersch H, Schweitzer L and Stuke J. Recombination process in $a$-Si:H: Spin-dependent photoconductivity. Physical Review B. 1983; 28:4678-4684. http://dx.doi.org/10.1103/ PhysRevB.28.4678
24. Kalevich VK, Shiryaev AYu, Ivchenko EL, Afanasiev MM, Egorov AYu, Ustinov VM et al. Hanle effect and spin-dependent recombination at deep centers in GaAsN Physica B. 2009; 404:4929-4932. http://dx.doi.org/10.1016/j. physb.2009.08.234

25. Zhao F, Balocchi A, Kunold A, Carrey J, Carre H, Amand $\mathrm{T}$ et al. Spin-dependent photoconductivity in nonmagnetic semiconductors at room temperature. Applied Physics Letters. 2009; 95:241104. http://dx.doi.org/10.1063/1.3273393

26. Peres ML, Chitta VA, Oliveira Junior NF, Maude DK, Rappl PHO, Ueta AY et al. Antilocalization of hole carriers in $\mathrm{Pb}_{1-x} \mathrm{Eu}_{x} \mathrm{Te}$ alloys in the metallic regime. Physical Review B. 2009; 79:085309-12. http://dx.doi.org/10.1103/ PhysRevB.79.085309

27. Grbic B, Ellenberger C, Ihn T, Ensslin K, Reuter D and Wieck AD. Magnetotransport in C-doped AlGaAs heterostructures. Applied Physics Letters. 2004; 85:2277-2279. http://dx.doi. org/10.1063/1.1781750

28. Spezia S, Adorno D P, Pizzolato N and Spagnolo B. Relaxation of electron spin during high-field transport in GaAs bulk. Journal of Statistical Mechanics. 2010; 11033. http://dx.doi. org/10.1088/1742-5468/2010/11/P11033 